\title{
OPINION AND TECHNICAL INDICATOR BASED OPTIMIZED DEEP LEARNING FOR PREDICTION OF STOCK MARKET
}

\author{
Jaymit B. Pandya \\ Ph.D. Research Scholar, Gujarat Technological University, Chandkheda, \\ Gandhinagar, Gujarat 382424, India \\ erpandyajaymit@gmail.com \\ Prof. (Dr.) Udesang K. Jaliya \\ Ph.D. Supervisor, Gujarat Technological University, Chandkheda, \\ Gandhinagar, Gujarat 382424, India, \& \\ Assistant Professor, Dept. of Computer Engineering, BVM Engineering College, \\ V.V. Nagar, Gujarat 388120, India \\ udayjaliya@gmail.com
}

\begin{abstract}
The prediction of stock movements has gained huge interest amongst the business world and academia. Due to globalization of advanced information technologies, the majority of peoples look forward in stock markets for earning huge returns. This paper presents stock market prediction using optimization driven deep model. Here, the review data of user and stock data are fed as an input to feature extraction. The extraction of features is done from the stock data and review data. From stock data, the technical indicators, like Absolute Price Oscillator (APO), Directional Movement Indicators (DMI), Simple Moving Average (SMA), WILLIAMS's \% R (WILLR), triangular moving average (TRIMA), and percent price oscillator (PPO) are extracted. From review data, the stemming and stop word removal methods are applied for extracting the irrelevant words. Then, the SentiWordNet is applied for extracting the review features. The obtained features are modeled in the feature vector and fed to deep convolutional long short-term memory (Deep-ConvLSTM) for stock prediction. Here, the training of Deep-ConvLSTM is performed by newly devised optimization technique, namely Rider based Elephant herd Optimization (Rider-EHO), which is obtained by integrating Rider Optimization Algorithm (ROA) and Elephant Herd Optimization (EHO). The proposed Rider-EHO-based Deep-ConvLSTM offered enhanced performance with smallest MSE of 0.003 and smallest RMSE of 0.059 .
\end{abstract}

Keywords: Stock market prediction, Deep learning, ConvLSTM, SentiWordNet, Elephant Herd Optimization.

\section{Introduction}

The prediction of stock market has gained huge interest from academic world and business. However, the extent to which the stock market is predicted remains a major challenge due to its complexity [12]. Stock market is considered as an imperative part in financial manoeuvre of contemporary societies. The evaluation of stock market index is a major interest amongst different stakeholders of the market. As per Efficient Market Hypothesis (EMH) [11], the prices of stock market reflect all the accessible information, and hence the prediction depends on information from different sources that can be classified into quantitative data, like turnover rate as well as historical prices, and qualitative data, like news, reports, social media posts and announcements. It is complex to handle qualitative data as they are unstructured and hence extraction of beneficial signals from the data is a challenging task [1]. The forecast of stock value had a huge interest amongst shareholders, managers and expertise because of the implication in the current age monetary trends. The forecast in stock market is termed as a complex and hesitant issue because of several assorted features that prevail in stock market, like unsteadiness, abnormality, clatter, trends in daily market and political control. The traders and investors rely on technical analysis that manoeuvres chronological or outdated and current stocks. Moreover, its complex to evaluate stock on basis of every day recorded stock data [10].

The precise forecast of erratic price refers understanding profitable shareholder in stock. The experiential assessment of stock from United stated of America (USA) has showed arithmetical consequence in return rate on investing stock market prediction by several data analysis. It has been depicted on possibility evidence for 
predicting stock price [13]. It is known that the classical research showed that its probable to forecast the stock prices using high profit by adapting current deep learning which is enhanced than the artificial intelligence technique. In Economics, there exist two techniques for predicting the stock prices which involves fundamental analysis and technical analysis. The technical analysis refers a technique in which the extraction of technical indicators is done by adapting numerical indicators like trading volume and stock price to forecast the stock price. The fundamental analysis refers a technique which relies on assessment of financial statements of company [14] [9]. In recent days, several techniques are devised to model and forecast the stock market which is devised using linear and nonlinear models. A sensible and precise prediction of stock rate movement can result into high financial benefits [15] [6].

For several decades, linear models are considered as source of classical forecasting models on monetary trades. The autoregressive moving average (ARMA) technique are comprehensively utilized for time series forecasting [16]. Auto-regressive heteroscedastic (ARCH), Moving average (MA) are considered as a huge acceptance amongst these statistical techniques [17]. They are extensively applied on various social, economic and engineering disciples. From many years, the random walk $(\mathrm{RW})$ is highly utilized and considered as a most leading statistical linear model in financial forecasting [6]. Hence, the classical works on predicting stock are devised on the basis of Efficient Market Hypothesis (EMH) and Random walk (RW) [18] [19]. To address the issue in prior years regarding stock prediction is complex task as different number of forecasting models are devised to forecast stocks. Several models rely on soft computing methods, which are generally termed as Artificial Neural Network (ANN), and Radial Basis Function Neural Network (RBFNN) [29]. The researchers have revealed several techniques to predict development of stock market with profits. The result of suggested neural network surpasses normal feed forward neural network in predicting the growth of market [20]. The interval evaluation of stock price disguises the advantage of adapting decision and inconsistency in harmonized stock. In [21], the prediction of stock price is advanced to predict the entire stock with lesser error [19] [10] [9].

The purpose is to devise a technique for prediction of stock to guarantee effectual stock prediction. At first, the input data are fed to feature mining that is devised on the basis of sentiment and opinion-based features along with technical indicators, such as APO, DMI, WILLR, SMA, TRIMA, and PPO. These technical indicators along with opinion-based features represent the whole features and used for the stock market prediction using the predictor. The features are fed to Deep-ConvLSTM model. The Deep-ConvLSTM model utilizes proposed RiderEHO algorithm, which is devised by combining ROA and EHO. Thus, the developed Rider-EHO based DeepConvLSTM offered effective performance using technical indicators.

The key contribution of the paper is:

- Proposed Rider-EHO based Deep-ConvLSTM model o forecast stock: Here, the proposed Rider-EHO based Deep-ConvLSTM is employed to predict stock movements. The training of Deep-ConvLSTM is carried out considering proposed Rider-EHO, and is devised by combining ROA and EHO.

The remaining paper is arranged as follows. Section 2 present conventional techniques devised on the basis of stock market prediction. Section 3 describes proposed Rider-EHO based Deep-ConvLSTM to predict stock market. Section 4 describes efficiency of proposed model and section 5 present the conclusion.

\section{Motivations}

The eight classical stock market prediction techniques are briefly illustrated along with its challenges and issues. These issues are taken as a motivation to devise a novel stock market prediction model.

\subsection{Literature review}

The eight classical stock prediction techniques are examined along with its issues. Xi Zhang et al. [1] devised a technique to forecast stock using movements of composite index. Here, the consistencies amongst several data sources were utilized and a multi-source multiple instance (M-MI) model was utilized for combining sentiments, and quantitative data in an inclusive model. For capturing news events, the event extraction and representation technique was utilized. Xi Zhang et al. [2] devised a technique for extracting the stocks using web news and sentiments of user from social media using coupled matrix and tensor factorization model. Here, the tensor was devised for fusing the heterogeneous data and captured the relationships amongst the events and sentiments of investors. Here, auxiliary matrices were utilized for stock quantitative feature matrix and the stock correlation matrix for decomposing the tensor. However, the method failed to consider behavioral finance for making decisions. Xi Zhang et al. [3] devised a technique based on tweets from Xueqiu which is a well-known Chinese Twitter, like social platform that are dedicated to investors. The features were analyzed based on the combined sentiment and discernment on stock relatedness and forecast the stock price movements by adapting nonlinear models. However, the method failed to consider time-series features. Xiongwen Pang, et al. [4] devised a neural network method for attaining stock prediction. Here, the data was attained using live stock market in real-time and the analytics were considered for analyzing the stocks. Moreover, the deep long short-term memory neural 
network (LSTM) and embedded layer with automatic encoder were considered to predict stock. However, the method failed to consider other datasets for stock movement prediction. S. Kumar Chandar [5] developed subtractive clustering-based adaptive neuro fuzzy approach to predict the prices of apple stock data. The model utilized a research data from 3rd Jan 2005 to 30th Jan 2015. Moreover, four technical indicators were devised for the analysis. These variables were considered as an input to neuro fuzzy system for predicting the stock prices. Nayak, et al. [6] devised an artificial chemical reaction optimization-based functional link network, termed ACFLN for predicting the stock market. Here, the effectiveness of the model was analyzed with real stock market prices. The method showed better performance in predicting the stock market. Xiaodong Li, et al. [7] devised Extreme Learning Machine (ELM) for predicting the stock market. The method attained elevated prediction accuracy and fast prediction speed. However, the reduction of CPU while keeping the prediction speed high is a major issue. M. M. Gowthul Alam and S. Baulkani [8] devised Multi-Kernel Support Vector Machine (MKSVM) that integrated global and local features of input data. Here, the parameter tuning method was devised with Fruit Fly Optimization (FFO) that is adapted to identify the movement of stock. However, the technique did not employ other stock database for evaluating performance.

\subsection{Challenges}

The major issues confronted by the classical stock prediction strategies are depicted below:

- The consideration of elevated correlations amongst the stock price and stock-based event on web for extracting events helps to guide the investment of stock. However, its capabilities in prediction are limited due to two issues. First is stock data accumulated from web is sparse, and second there is a deficiency of technique which can qualitatively analyze the stock price as it is complex to discover if the event causes negative impact or positive impact on stock price.

- Polynomial function and Kernel function Gaussian function are used for constructing multi-kernel function for predicting stock price. However, in realistic applications, efficiency of MSVM relies on certain attributes, which is complex to choose due to arbitrariness of calculation procedure [8].

- It is complex to acknowledge the classical optimization techniques, like genetic algorithm and particle swarm optimization for implementing and discovering the global optimum solution [8].

- ANN gains the interest of several authors to devise ANN-assisted forecasting techniques for predicting stock. However, the ANN assisted forecasting techniques suffered from huge computation complexion as it requires several layers and huge neurons in network layer [6] [22].

- In prior research [5], a deep learning technique is utilized considering five stock data. However, the estimation of prices is complex as there exist several things considered in the training as ranges of prices varies with each other and hence training is a non-trivial task [9][22].

\section{Proposed Rider-EHO-assisted Deep-ConvLSTM for Predicting Stock Market}

The rapid extension of stock market has gained the interest amongst several peoples for carrying out the detailed and scientific research, which attempts to describe rules by which stock market function that are apparently deviating. The investor tries to mine features as by adapting a promising indicator in order to compute stock market trends. The prior research is based on EMH and RW theory, but these techniques are unable to offer optimal performance. Thus, this research devises a novel optimization driven deep model. The major contribution is to forecast future market using past and present market status. At first, the input stock data and user review data are fed to feature extraction that is based on the sentiment and opinion-based features along with technical indicators, such as APO, DMI, SMA, WILLR, TRIMA, and PPO. The technical indicators are the stock data that considerably donates in forecasting a stock. These technical indicators along with opinion-based features represent the complete features and are utilized for predicting the stock market using Deep-ConvLSTM. The highly imperative features of data are subjected to prediction wherein Deep-ConvLSTM model is adapted. The training of Deep-ConvLSTM model is carried out with proposed Rider-EHO that is devised by integrating ROA and EHO. Fig. 1 present's schematic view of prediction model using proposed Rider-EHO based Deep-ConvLSTM.

\subsection{Acquisition of time series data}

Time series is an imperative class of temporal data objects and it is generated using financial and scientific applications. The time-series data is an accumulation of observations made in a chronological way. Here, the time series data of stock market and review of user is considered which helps to predict the trends in market with respect to time. 


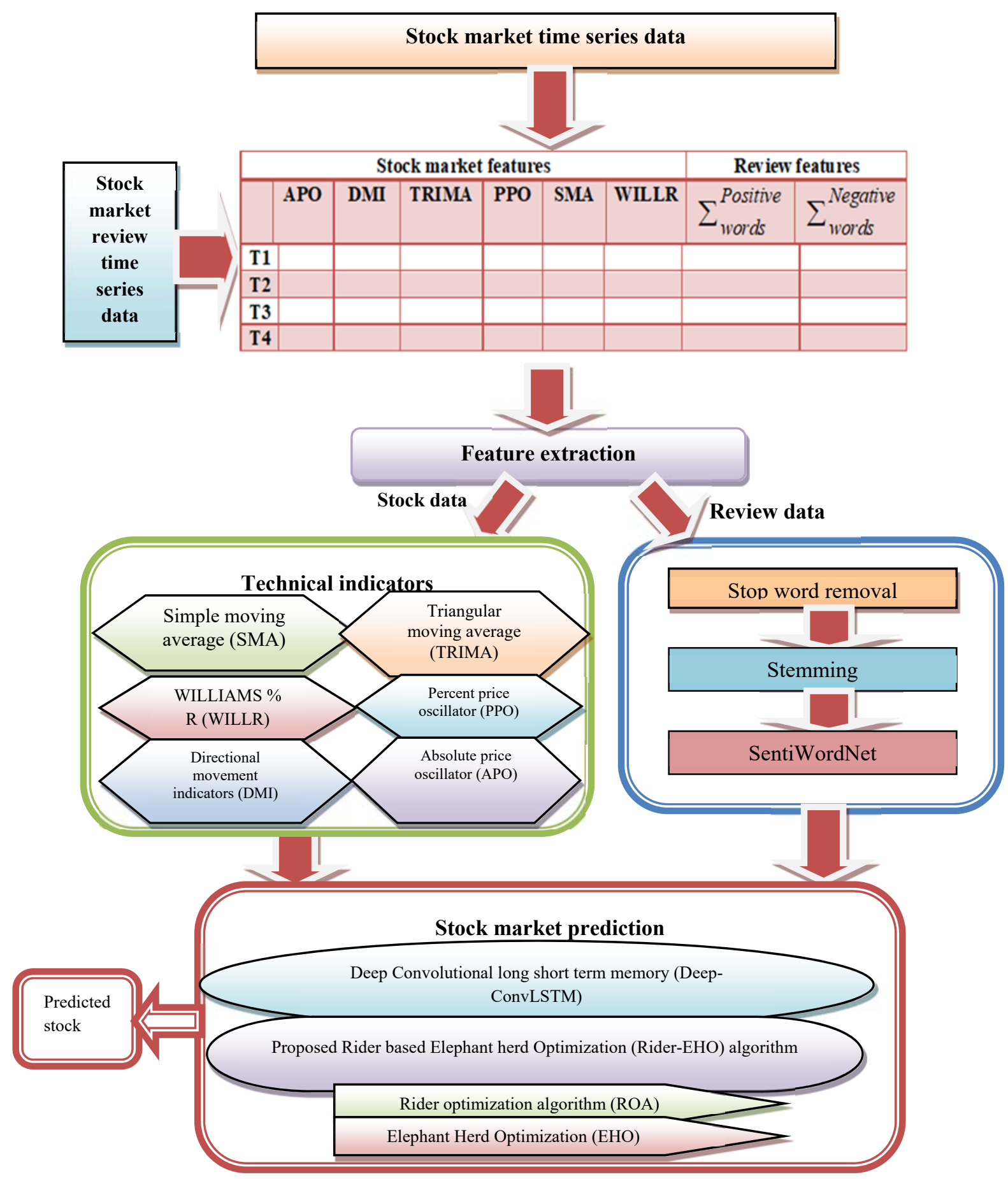

Fig. 1. Architecture of proposed Rider-EHO-based Deep ConvLSTM for stock market prediction

\subsubsection{Reading of input stock market data}

The prediction of stock market is carried out by adapting the inputted stock data. Here, the prices of stocks are examined for each day on the basis of time-series data that assist to make precise prediction in stock market. Assume the stock data as $K$ accumulated from stock market database $G$. The stock market data $K$ is utilized to attain the prediction by extracting the imperative features using technical indicators.

\subsubsection{Read the input review data}

Consider input data expressed as $B$ with various attributes, and modeled as,

$$
B=\left\{B_{c, d}\right\} ;(1 \leq c \leq b)(1 \leq d \leq a)
$$


where, $B_{c d}$ represent user review data present in dataset $B$ with $c^{\text {th }}$ attribute in $d^{\text {th }}$ data. Here, $a$ represent total data and $b$ is count of attributes. Each data is subjected to feature mining phase, in which stopping word removal, stemming and SentiWordNet is applied for extracting the review based features.

\subsection{Feature extraction using inputted stock market data and reviews}

Once the time series data $G$ and $B$ is obtained, the features are obtained with time series data. The mining of features assures effectual prediction of stock market. For extracting features from stock data, the technical indicators are adapted whereas for extracting review-based features, the SentiWordNet is applied.

\subsubsection{Technical indicator for extracting stock market features}

The technical indicators are considered as an imperative part in mining features using data which facilitates effectual prediction. The technique adapts six technical indicators for extracting the stock features. Here, the stock market data $K$ is employed to mine the technical indicator features that involve APO, DMI, SMA, WILLR, TRIMA, and PPO. The technical indicators indicate a series of data points which are mined by adapting the function using the price data at study period and time. The technical indicator covers different feature kinds, namely price change, buy and sell signals, stock trend discovery, and noise removal and data smooth features. The technical indicators which are mined using the input stock market data $K$ is portrayed below:

a) WILLIAMS \% R (WILLR):

It is particularly known as Williams \%R that helps to discover the closing price of day on the basis of past ten day's transaction. Williams \% R, also termed as Williams Percent Range, which is a kind of momentum indicator that ranges between 0 and -100 and helps to compute oversold and overbought levels. The Williams \%R can be utilized for determining the exit and entry points in market and offer an insight in strength and weakness of stock. The WILLR is formulated as,

$$
W_{l l}=\frac{b}{c} * 100
$$

where, $b$ symbolizes the highest closed, and $c$ represent the highest price. The WILLR feature is indicated by $F_{l}$.

b) Simple Moving Average (SMA):

It refers an AMA computed by adding current prices and then divides the figure using a time periods count. It is the simplest moving average to construct and is an average price over particular period. The SMA are utilized to detect the direction of trend. Here, if the SMA moves up then trend is up and if the SMA moves down, then the trend is down. It is evaluated on the basis of average price at a particular time period $r$. SMA is computed as,

$$
G=\frac{1}{d} \sum_{j=0}^{d-1} Y_{t-j}
$$

where, $Y_{t}$ indicate closing price on day $t$, and $d$ represent input window length. The SMA feature is represented as $F_{2}$.

c) Triangular Moving Average (TRIMA)

The TRIMA indicates an average of prices and places weight on middle price of time period. It is a WMA that smoothes the market noise and reveals trends of market in a clear manner. The indicator helps to filter the fluctuations of market by averaging the price values with respect to time. The TRIMA is formulated as,

$$
T R I M A=\frac{S M A_{1}+S M A_{2}+\ldots+S M A_{N}}{N}
$$

where, $N$ is count of stocks. The TRIMA feature is expressed as $F_{3}$.

d) Directional Movement Indicators (DMI)

The DMI is an indicator that recognized the direction in which the assets price is moving. The indicator performs those by comparing the highs and lows by drawing two lines as positive directional movement line $(+\mathrm{DMt})$ and a negative directional movement line (-DMt). This indicator helps the traders to access direction of trend and DMI is expressed as,

$$
\begin{aligned}
& +D M t=[+D M t-1-(+D M t-1 / n)] \\
& -D M t=[-D M t-1-(-D M t-1 / n)]
\end{aligned}
$$

where, $+D M t$ is $+D M$ at a given time, $t$ and $+D M t-1$ is $+D M$ at a given time $t-1,-D M t$ is $-D M$ at a given time, $t$ and $-D M t-1$ is $-D M$ at a given time $t-1$. The DMI feature is expressed as $F_{4}$ 
e) Absolute Price Oscillator (APO)

The APO refers the difference amongst two EMA and is modeled as an absolute value. The APO is devised using absolute differences amongst two moving averages of varying lengths fast and slow moving average. The positive indicator value represents an upward movement and negative signal represent downward trend. Here, the APO is formulated as,

$$
\mathrm{APO}=\text { Fast Exponential Moving Average-Slow Exponential Moving Average }
$$

Thus, the APO feature is represented as $F_{5}$.

f) Percent Price Oscillator (PPO)

The PPO is devised on the basis of differences amongst two moving averages by altering lengths fast SMA. The PPO computes the difference of two averages divided by slower of two moving averages, and it tends to normalize values. The PPO is a nine-day exponential moving average, reduced and then divided by 26-day exponential moving average, which is expressed as,

$$
P P O=\frac{E M A_{9 \_ \text {day }}-E M A_{26 \_d a y}}{E M A_{26 \_d a y}}
$$

where, EMA is exponential moving average of stocks closing price. The PPO feature is expressed as $F_{6}$.

\subsubsection{Extraction of review-based features}

Before extracting the review-based features, the review data should be pre-processed by applying stemming and stop word removal in order to provide effective features. The review database consists of unnecessary words that may impact feature extraction. Thus, the removal of stop word and stemming is performed to remove inconsistent words by refining the reviews. Here, the input user review data $B$ is employed, which is briefly illustrated below.

i) Stop word removal:

The stop words indicate a word that does not hold any data. The removal of stop word is a procedure for eliminating stop words using large textual data. Here, the non-informative words are removed to minimize noise present in data. The elimination of stop words are utilized for saving huge space and make processing fast by attaining effectual outcomes. In this model, the stop words, like nouns, verbs are removed from review data.

ii) Stemming:

The stemming procedure is utilized for transforming words to stem. In huge documents, different words are used, which depicts similar concept. The imperative method utilized for minimizing words to root is known as stemming. For example, agreeing, agree and disagree belongs to agree. The practice of stemming is condensed and easy to utilize and is precise.

Thereafter, the feature extraction of condensed user review data is done using SentiWordNet. Here, the opinion-based feature obtained from the opinions of user regarding a product is considered. The SentiWordNet is applied on the keywords of user review data.

SentiWordNet [27] is adapted as a lexical resource to mine the opinions of user. SentiWordNet assigns each synset of WordNet considering two sentiment numerical words, like positive Pos(s), and negative Neg(s). Different words have different polarities with lemma to identify different word senses. The SentiWordNet is adapted for discovering polarity of a review. SentiWordNet offers the automated annotation of synsets on the basis of positivity", "negativity", and "neutrality". Hence, the SentiWordNet is adapted to discover positivity, and negativity, of words contained in review data. Thus, the SentiWordNet is modeled as,

$$
S(W)=\{P, N\}
$$

After the application of SentiWordNet, two types of review-based features are extracted that involves positive words set and negative words set and represented as $F_{7}$ and $F_{8}$ and is formulated as,

$$
F_{7}=\sum_{i=1}^{n} P_{i}
$$

where, $n$ signifies count of words in a review such that $1 \leq i \leq n, P_{i}$ denote that the $i^{\text {th }}$ word is positive.

$$
F_{8}=\sum_{i=1}^{n} N_{i}
$$

where, $n$ signifies count of words in a review such that $1 \leq i \leq n, N_{i}$ denote that the $i^{t h}$ word is negative. Thus, the stock data features and the review-based features are extracted, which are collected in feature vector $F$ and 
generated feature vector is subjected to proposed Rider- EHO based Deep-ConvLSTM for effective prediction. The feature vector is formulated as,

$$
F=\left\{F_{1}, F_{2}, F_{3}, F_{4}, F_{5}, F_{6}, F_{7}, F_{8}\right\}
$$

where, $F_{1}, F_{2}, F_{3}, F_{4}, F_{5}, F_{6}$ represent stock data features obtained from technical indicators such as WILLR, SMA, TRIMA, DMI, APO and PPO and $F_{7}, F_{8}$ signifies review-based features that involves set of positive words and negative words.

\subsection{Stock prediction with proposed Rider-EHO based Deep-ConvLSTM model}

The prediction of stock market is done using proposed Rider-EHO based Deep-ConvLSTM. For effective prediction, the Deep-ConvLSTM is utilized, which helps to discover optimum weights-biases using proposed Rider-EHO algorithm. Hence, the prediction of stock market is performed with Deep-ConvLSTM, which acquires effectual features as an input. The training of Deep-ConvLSTM is done by utilizing proposed Rider-EHO, which is obtained by combining EHO [24] and ROA [23]. The Deep-ConvLSTM is able to predict future using inputs and past states based on the neighbors. The Deep-ConvLSTM offers effectual feature patterns with huge transitional kernel. For performing effective forecast, the Deep-ConvLSTM is set as encoding and forecasting layers. The brief explanation of Deep-ConvLSTM architecture and training of Deep-ConvLSTM with proposed Rider-EHO are given below.

\subsubsection{Architecture of Deep-ConvLSTM model}

The obtained feature vector $F$ is subjected to Deep-ConvLSTM to attain prediction of stock market. As the DeepConvLSTM [26] acquires additional benefits in contrast to other classifiers like Deep-ConvLSTM is effectual to attain forecast of stock market on the basis of memory cell of classifier. Deep-ConvLSTM utilizes past states and input corresponding to its neighbors for predicting future states with Hadamard product ${ }^{\circ}$ and Convolutional operator*. The Deep-ConvLSTM offers effectual stock prediction by adapting high transitional kernel, and structure of Deep-ConvLSTM is established using forecasting and encoding layers. Fig. 2 displays structural design of Deep-ConvLSTM classifier.

The Deep-ConvLSTM comprises input $\mathrm{P}_{1}$, cell $\mathrm{M}_{1}$ and output $\mathrm{O}_{1}$, weight $\omega_{\mathrm{r}, \mathrm{s}}$ which is fully connected to Pl. Here, the input $\mathrm{P}_{1}$ and working memory $\mathrm{O}_{1-1}$ are integrated for learning the forget gate. In ConvLSTM each attribute in forget gate tensor discovers to which extent the long-term memory elements in $[0,1]$ exists. Here, the highest value 1 refers completely saved and smallest value 0 refers completely forgotten. The mathematical representation of Deep-ConvLSTM is given below.

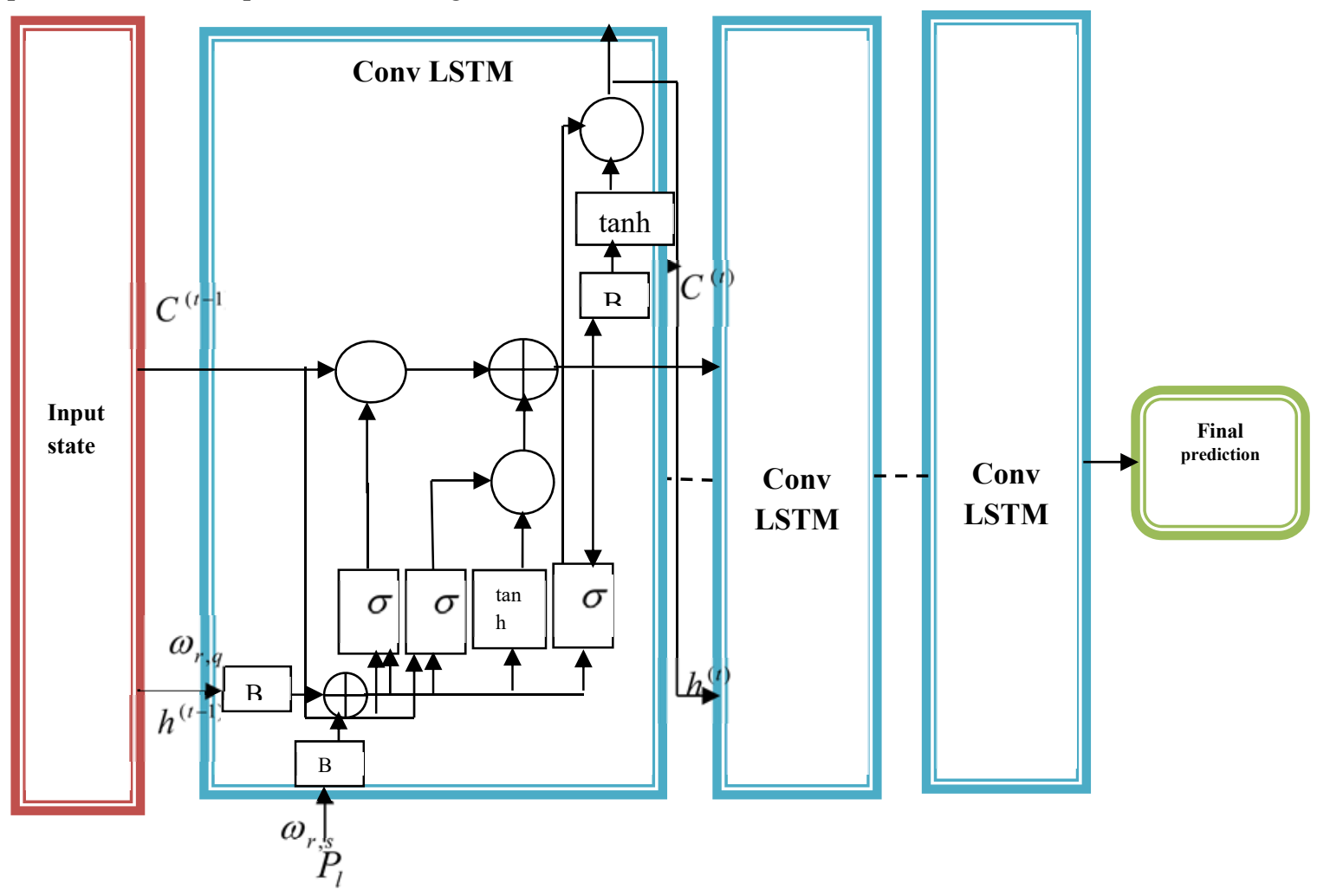

Fig. 2. Structural design of Deep-ConvLSTM classifier 
Initially, the classifier showed in Fig. 2. is employed for leaning the forget gate Ds which is formulated as,

$$
D_{s}=\sigma\left(\omega_{r, s} * P_{l}+\omega_{m, s} * O_{l-1}\right)+n_{s}
$$

where, $\sigma$ indicate sigmoid function, $\omega_{\mathrm{r}, \mathrm{s}}$ and $\omega_{\mathrm{m}, \mathrm{s}}$ indicates weights associated between the conv layers, $O_{l-1}$ symbolize working memory, and $P_{l}$ is input.

Secondly, the extraction of information can be learned from $P_{l}$ which is the candidate memory $D$ of long-term memory, which is expressed as,

$$
D_{s}=\tanh \left(\omega_{r} * P_{l}+\omega_{m} * O_{l-1}+n\right)
$$

Third step is combination of above two steps. The aim is to forget the memory which is not received and save the useful part of input. Thereafter, the update of long-term memory $H_{l}$ is generated, which is illustrated as,

$$
\begin{gathered}
D_{k}=\sigma\left(\omega_{r, k} * P_{l}+\omega_{m, k} * O_{l-1}+n_{k}\right) \\
H_{l}=D_{s} \circ H_{l-1} \circ D_{k} \circ D
\end{gathered}
$$

Fourth is update of working memory. The network requires the learner to focus the long term memory and makes more sense in current step. Hence the Leaning of focus vector $D_{q}$ is expressed as,

$$
D_{q}=\sigma\left(\omega_{r, q} \times P_{l}+\omega_{m, q} * O_{l-1}+n_{q}\right)
$$

Fifth, the working memory is evaluated. In other words, the network focus on element where attention vector is 1 and ignores the element whose attention vector is 0 and is expressed as,

$$
O_{l}=D_{o} \circ \tanh \left(H_{k}\right)
$$

The output obtained from the Deep-ConvLSTM classifier is denoted by $O$.

\subsubsection{Training of Deep-ConvLSTM using Proposed Rider-EHO algorithm}

The prediction of stock market is attained using Deep-ConvLSTM classifier, whose training is performed by proposed Rider-EHO algorithm. Here, the proposed Rider-EHO algorithm is combination of ROA and EHO. The properties of ROA are inherited by the EHO algorithm to improve the efficiency of predicting the stock market. The developed Rider-EHO is highly effectual to perform stock prediction with limited memory. EHO is inspired from behavior herding. These behaviors can be expressed in operators, which are clan updating operator and separating operator. In EHO [24], the elephants from each clan are updated by present position and clan updating operator. Then, it utilizes separating operator, which poses the ability to improve population diversity by solving global optimization based tasks. Furthermore, the elephant's habitation can be utilized for solving optimization issues. On the other hand, ROA [23] is inspired from rider behavior, who tends to travel a common target to become a winner. In this method, the riders are chosen from the total riders of each group. Each group undergoes several strategies for attaining the target. In addition, the ROA is highly effective and follows fictional computing to solve optimization problems, but poses less convergence and are extremely sensitive to hyper parameters. Thus, the combination of ROA and EHO can help to generate global optimal solutions. The steps of proposed RiderEHO-based Deep-ConvLSTM are examined as:

Step 1) Initialization: The preliminary step is solution initiation, which is given as,

$$
S=\left\{S_{1}, S_{2}, \cdots, S_{h}, \cdots S_{i}\right\} ; 1 \leq h \leq i
$$

where, $i$ denotes total number of solutions and $S_{h}$ signifies $h^{\text {th }}$ solution

Step2) Discovery of Error: The best solution is determined with error, and is modeled as minimization issue and hence, solution with least MSE is chosen as an optimal solution and MSE is expressed as,

$$
M S_{e r r}=\frac{1}{g} \sum_{f=1}^{g}\left[O_{f}-O\right]^{2}
$$

where, $O_{f}$ denote expected output and $O$ signifies predicted output, $g$ denote count of number of inputs, where $1<f$ $\leq g$.

Step3) Determination of weights: In order to solve all the optimization issues, the EHO is employed. As per EHO [24], the update is given by, 


$$
S_{d, e}^{b+1}=S_{d, e}^{b}+\beta\left(S_{d}^{b e s t}-S_{d, e}^{b}\right) \times c
$$

where, $S_{d, e}^{b+1}$ and $S_{d, e}^{b}$ represent new and old elephant position e in clan d, $\beta$ signifies constant ranging between 0 and $1, \mathrm{c}$ is an arbitrary number between 0 and $1, S_{d}^{\text {best }}$ is fittest elephant individual in clan d.

$$
\begin{gathered}
S_{d, e}^{b+1}=S_{d, e}^{b}+\beta c S_{d}^{\text {best }}-\beta c S_{d, e}^{b} \\
S_{d, e}^{b+1}=S_{d, e}^{b}(1-\beta c)+\beta c S_{d}^{\text {best }}
\end{gathered}
$$

In ROA [23], the bypass rider has the ability to bypass the common path without utilizing path of leading rider, and thus position of bypass rider is provided in random basis and is formulated as,

$$
S_{d, e}^{b+1}=\delta\left[S_{\alpha, e}^{b} * \eta_{e}+S_{\sum e}^{b} *\left(1-\eta_{e}\right)\right]
$$

where, $\delta$ is an arbitrary number ranging between 0 and $1, \eta$ signifies arbitrary number ranging between 1 and $\mathrm{R}$.

Let $\alpha=\mathrm{d}$ then equation (24) can be rewritten as,

$$
\begin{gathered}
S_{d, e}^{b+1}=\delta S_{d, e}^{b} \eta_{e}+\delta S_{\sum e}^{b}\left(1-\eta_{e}\right) \\
\delta S_{d, e}^{b} \eta_{e}=S_{d, e}^{b+1}-\delta S_{\sum e}^{b}\left(1-\eta_{e}\right) \\
S_{d, e}^{b}=\frac{S_{d, e}^{b+1}-\delta S_{\sum_{e} b}^{b}\left(1-\eta_{e}\right)}{\delta \eta_{e}}
\end{gathered}
$$

Substituting equation (27) in equation (23),

$$
\begin{gathered}
S_{d, e}^{b+1}=\frac{S_{d, e}^{b+1}-\delta S_{\sum e}^{b}\left(1-\eta_{e}\right)}{\delta \eta_{e}}(1-\beta c)+\beta c S_{d}^{b e s t} \\
S_{d, e}^{b+1}-\frac{S_{d, e}^{b+1}(1-\beta c)}{\delta \eta_{e}}=\frac{-\delta S_{\sum e}^{b}\left(1-\eta_{e}\right)}{\delta \eta_{e}}(1-\beta c)+\beta c S_{d}^{\text {best }} \\
\frac{S_{d, e}^{b+1} \delta \eta_{e}-S_{d, e}^{b+1}(1-\beta c)}{\delta \eta_{e}}=\frac{-\delta S_{\sum e}^{b}\left(1-\eta_{e}\right)}{\delta \eta_{e}}(1-\beta c)+\beta c S_{d}^{b e s t}
\end{gathered}
$$

The update equation of proposed Rider-EHO algorithm is represented as,

$$
S_{d, e}^{b+1}=\frac{\delta \eta_{e}}{\delta \eta_{e}-(1-\beta c)}\left[\frac{-\delta S_{\sum_{e}}^{b}\left(1-\eta_{e}\right)}{\delta \eta_{e}}(1-\beta c)+\beta c S_{d}^{b e s t}\right]
$$

\begin{tabular}{|l|l|}
\hline SI. No & \multicolumn{1}{|c|}{ Pseudo code of proposed Rider-EHO } \\
\hline 1 & Input: Population $S$, iteration $b$ \\
\hline 2 & Output: Best solution $S^{*}$ \\
\hline 3 & Begin \\
\hline 4 & Initialize generation counter $b=1$, population and max generation counter $b_{\max }$ \\
\hline 5 & While $b<b_{\max }$ do \\
\hline 6 & Sort population with error using Eq. (20) \\
\hline 7 & Execute clan updating operator \\
\hline 8 & Execute separating operator \\
\hline 9 & Evaluate population using new update solution considering Eq. (26) \\
\hline 10 & $b=b+1$ \\
\hline 11 & End while \\
\hline 12 & End \\
\hline
\end{tabular}

Table 1. Pseudo code of proposed Rider-EHO 
By adapting herding behavior of elephants with ROA, the performance of stock market prediction is enhanced. The moment estimation of proposed Rider-EHO algorithm provides the enhancement in prediction of stock prices. Step 4) Error computation for update solutions: The error is recomputed, in which weights associated to minimal error is adapted for Deep-ConvLSTM training.

Step 5) Terminate: The best weights are generated repeatedly till maximum iteration is accomplished. Table 1 displays pseudo code of proposed Rider-EHO.

\section{Results and Discussion}

The efficiency of proposed Rider-EHO based Deep-ConvLSTM is computed using MSE and RMSE. The assessment is carried out by altering training data from $50 \%$ to $90 \%$.

\subsection{Experimental setup}

The implementation of developed model is carried out in MATLAB using i3 processor and 2GB RAM.

\subsection{Dataset description}

The stock market dataset is utilized to perform prediction of stock market, which comprises stock data of different companies taken from the year 2000-2020. In this analysis, two different datasets are utilized for performing stock market prediction, which is Reliance Communications, and Relaxo Footwear.

Dataset 1: The dataset-1 consists of stock data using Reliance communications with dailybasis considering thestart date of 1 January 2019 to 1 December 2020.

Dataset-2:The dataset-2 consists of stock data using Reliance communications with monthly basis considering the start date of 1 January 2000 to 1 December 2020.

Dataset-3: The dataset-3 consists of stock data using Reliance communications with yearly basis considering the start date of 2000 to 2020 .

Dataset 4: The dataset-1 consists of stock data using Relaxo Footwear with daily basis considering the start date of 1 January 2019 to 1 December 2020.

Dataset-5: The dataset-2 consists of stock data using Relaxo Footwear with monthly basis considering the start date of 1 January 2000 to 1 December 2020.

Dataset-6: The dataset-3 consists of stock data using Relaxo Footwear with yearly basis considering the start date of 2000 to 2020 .

\subsection{Performance measures}

The evaluation metrics utilized for evaluating efficiency of proposed model in contrast to another model is briefed below.

MSE: The MSE definition is already described in Eq. (20).

RMSE: It refers square root of difference amongst actual and evaluated value, and is formulated as,

$$
R M S E=\sqrt{\left[\frac{\sum_{f=1}^{g}\left[O_{f}-O\right]^{2}}{G}\right]}
$$

\subsection{Experimental results}

The methods taken for comparison involves FFO+MKSVM [8], Genetic algorithm-based convolution neural network (GA-CNN) [28], M-MI model [1], Deep LSTM [4], EHO [24], and proposed Rider-EHO based DeepConvLSTM.

The assessment of techniques using Reliance communications and Relaxo Footwear data considering daily, monthly, and yearly basis are evaluated. The assessment is carried out by varying training data from $50 \%$ to $90 \%$.

\subsubsection{Assessment with dataset-1}

Fig. 4 presents the assessment of techniques using Reliance communication dataset considering daily basis scenario with MSE and RMSE. The assessment of techniques with MSE is depicted in Fig. 4a. For 50\% training data, the MSE measured by FFO+MKSVM, GA-CNN, M-MI model, Deep LSTM, EHO, and proposed RiderEHO based Deep-ConvLSTM are 427.076, 34.185, 0.328, 0.197, 0.044, and 0.018. Likewise, for $90 \%$ data, the MSE measured by FFO+MKSVM, GA-CNN, M-MI model, Deep LSTM, EHO, and proposed Rider-EHO based Deep-ConvLSTM are 86.386, 0.377, 0.129, 0.033, 0.013, and 0.003. The assessment of techniques with RMSE is depicted in Fig. 4b. For 50\% training data, the RMSE measured by FFO+MKSVM, GA-CNN, M-MI model, Deep LSTM, EHO, and proposed Rider-EHO based Deep-ConvLSTM are 20.666, 5.847, 0.573, 0.444, 0.211, and 0.133 . Likewise, for $90 \%$ data, the RMSE measured by FFO+MKSVM, GA-CNN, M-MI model, Deep LSTM, EHO, and proposed Rider-EHO based Deep-ConvLSTM are 9.294, 0.614, 0.360, 0.182, 0.116, and 0.059. 


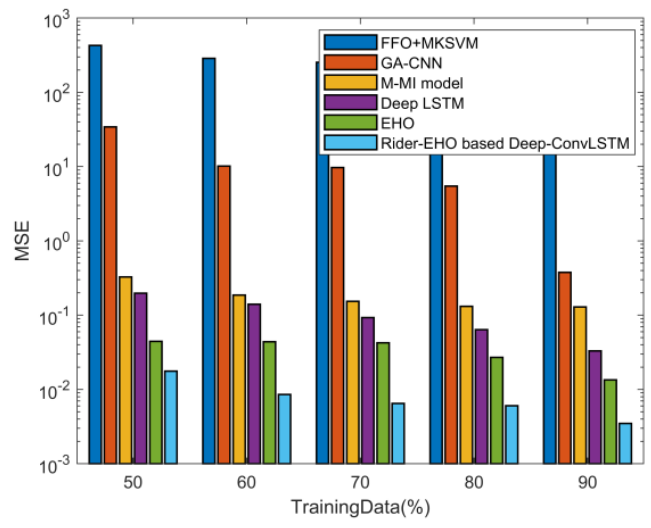

(a)

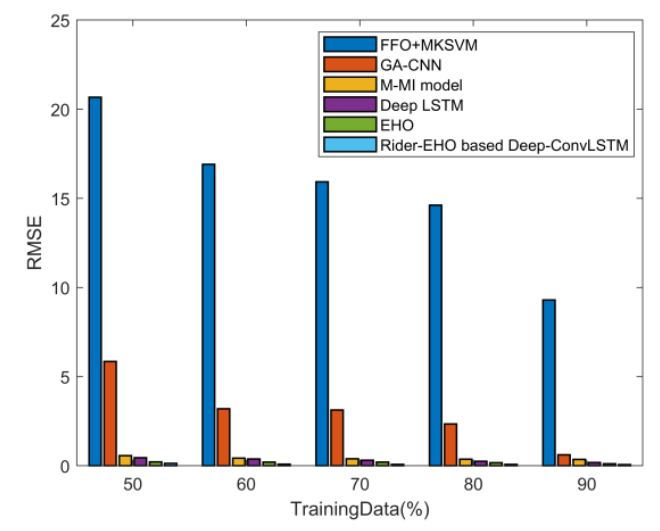

(b)

Fig. 4. Assessment of techniques using Reliance communication dataset considering daily basis scenario with a) MSE b) RMSE

4.4.2 Assessment with dataset-2

Fig. 5 presents the assessment of techniques using Reliance communication dataset considering monthly basis scenario with MSE and RMSE. The assessment of techniques with MSE is depicted in Fig. 5a. For 50\% training data, the MSE measured by FFO+MKSVM, GA-CNN, M-MI model, Deep LSTM, EHO, and proposed RiderEHO based Deep-ConvLSTM are 8363.030, 3947.858, 3821.064, 1518.829, 840.559, and 695.458. Likewise, for $90 \%$ data, the MSE measured by FFO+MKSVM, GA-CNN, M-MI model, Deep LSTM, EHO, and proposed Rider-EHO based Deep-ConvLSTM are 779.248, 440.134, 150.028, 117.319, 83.308, and 44.435. The assessment of techniques with RMSE is depicted in Fig. 5b. For 50\% training data, the RMSE measured by FFO+MKSVM, GA-CNN, M-MI model, Deep LSTM, EHO, and proposed Rider-EHO based Deep-ConvLSTM are 91.450, $62.832,61.815,38.972,28.992$, and 26.372 . Likewise, for $90 \%$ data, the RMSE measured by FFO+MKSVM, GA-CNN, M-MI model, Deep LSTM, EHO, and proposed Rider-EHO based Deep-ConvLSTM are 27.915, $20.979,12.249,10.831,9.127$, and 6.666.

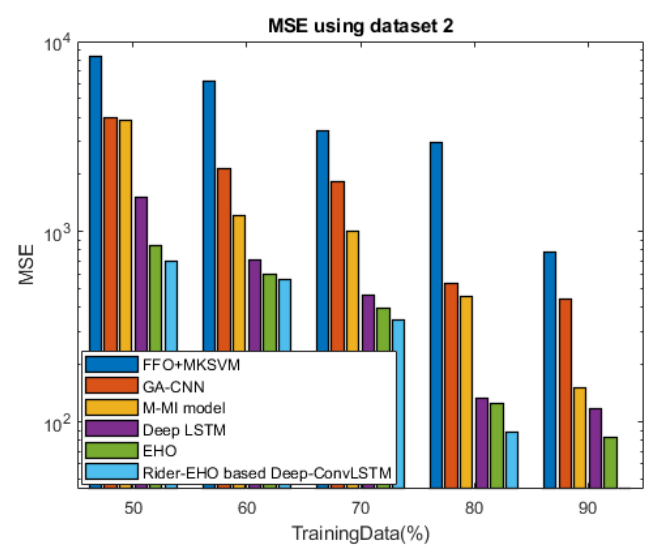

(a)

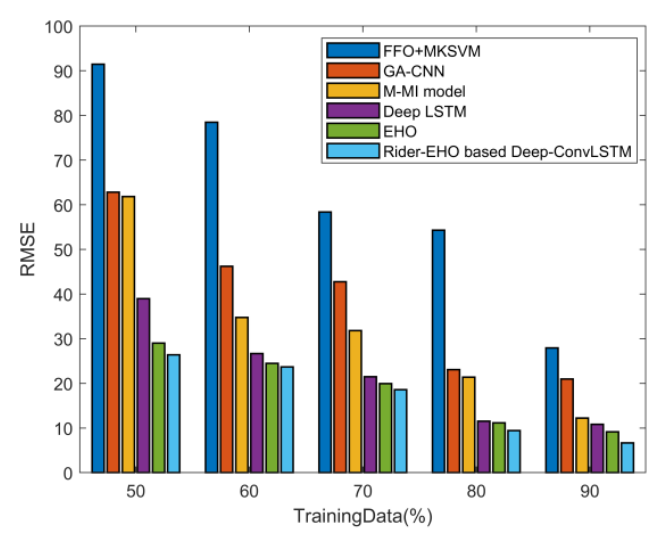

(b)

Fig. 5. Assessment of techniques using Reliance communication dataset considering monthly basis scenario with a) MSE b) RMSE

\subsubsection{Assessment with dataset-3}

Fig. 6 presents the assessment of techniques using Reliance communication dataset considering yearly basis scenario with MSE and RMSE. The assessment of techniques with MSE is depicted in Fig. 6a. For 50\% training data, the MSE measured by FFO+MKSVM, GA-CNN, M-MI model, Deep LSTM, EHO, and proposed RiderEHO based Deep-ConvLSTM are 113473.704, 91563.270, 50805.488, 18651.930, 5352.844, and 1943.666. Likewise, for $90 \%$ data, the MSE measured by FFO+MKSVM, GA-CNN, M-MI model, Deep LSTM, EHO, and proposed Rider-EHO based Deep-ConvLSTM are 8997.309, 699.406, 413.183, 322.455, 253.156, and 50.396. The assessment of techniques with RMSE is depicted in Fig. 6b. For 50\% training data, the RMSE measured by FFO+MKSVM, GA-CNN, M-MI model, Deep LSTM, EHO, and proposed Rider-EHO based Deep-ConvLSTM are $336.859,302.594,225.401,136.572,73.163$, and 44.087. Likewise, for $90 \%$ data, the RMSE measured by FFO+MKSVM, GA-CNN, M-MI model, Deep LSTM, EHO, and proposed Rider-EHO based Deep-ConvLSTM are $94.854,26.446,20.327,17.957,15.911$, and 7.099 . 


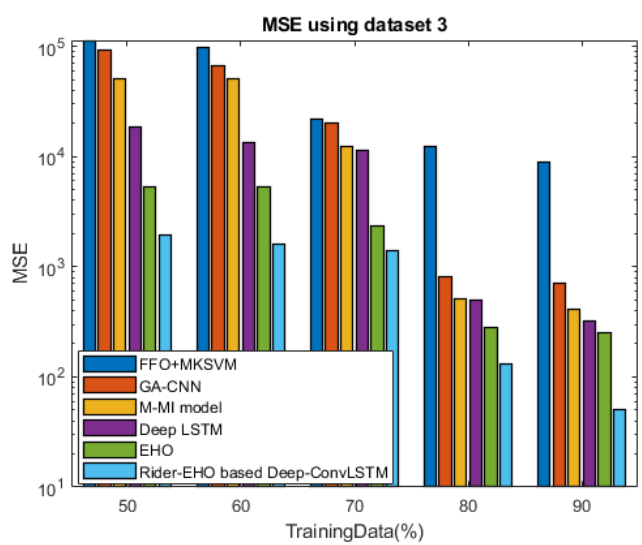

(a)

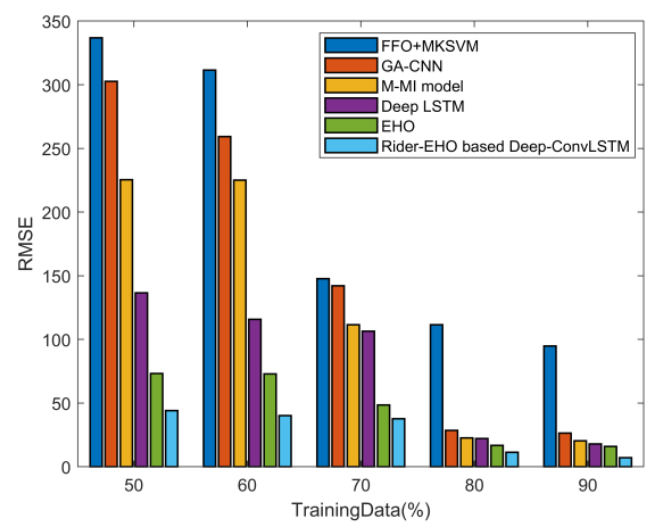

(b)

Fig. 6. Assessment of techniques using Reliance communication dataset considering yearly basis scenario with a) MSE b) RMSE

\subsubsection{Assessment with dataset-4}

Fig. 7 presents the assessment of techniques using Relaxo Footwear dataset considering daily basis scenario with MSE and RMSE. The assessment of techniques with MSE is depicted in Fig. 7a. For 50\% training data, the MSE measured by FFO+MKSVM, GA-CNN, M-MI model, Deep LSTM, EHO, and proposed Rider-EHO based DeepConvLSTM are 311887.521, 206725.754, 2588.969, 2186.087, 2148.638, and 386.354. Likewise, for $90 \%$ data, the MSE measured by FFO+MKSVM, GA-CNN, M-MI model, Deep LSTM, EHO, and proposed Rider-EHO based Deep-ConvLSTM are 127871.562, 15644.667, 170.773, 142.793, 86.504, and 3.731. The assessment of techniques with RMSE is depicted in Fig. 7b. For 50\% training data, the RMSE measured by FFO+MKSVM, GA-CNN, M-MI model, Deep LSTM, EHO, and proposed Rider-EHO based Deep-ConvLSTM are 558.469, $454.671,50.882,46.756,46.353$, and 19.656. Likewise, for $90 \%$ data, the RMSE measured by FFO+MKSVM, GA-CNN, M-MI model, Deep LSTM, EHO, and proposed Rider-EHO based Deep-ConvLSTM are 357.591, $125.079,3.068,11.950,9.301$, and 1.931 .

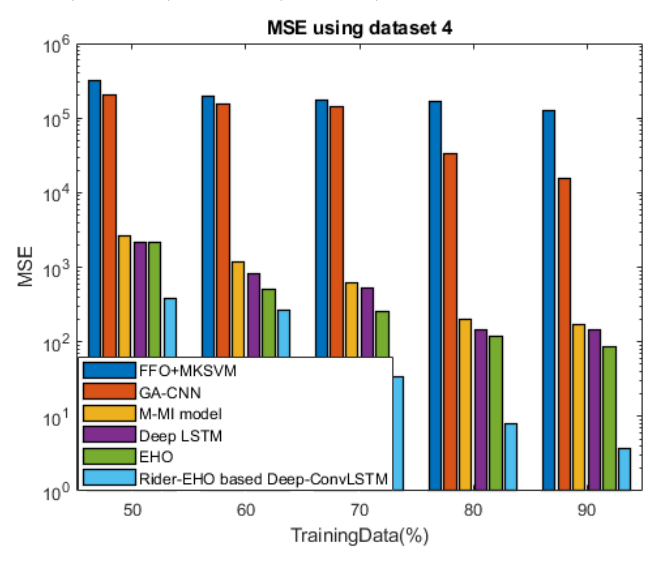

(a)

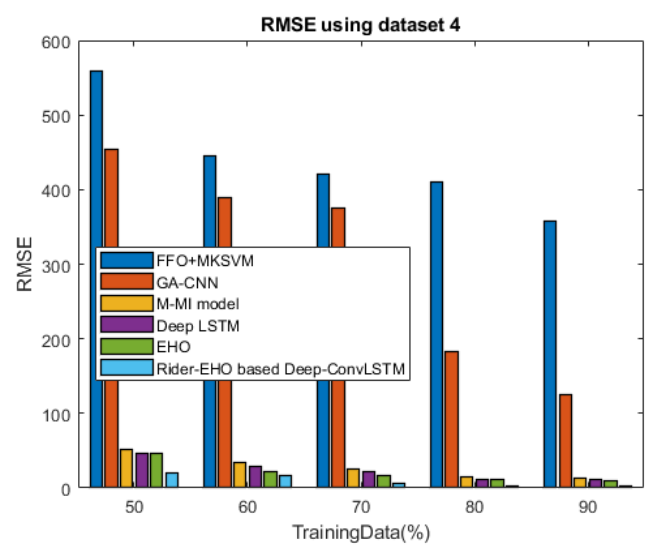

(b)

Fig. 7. Assessment of techniques using Relaxo Footwear dataset considering daily basis scenario with a) MSE b) RMSE

\subsubsection{Assessment with dataset-5}

Fig. 8 presents the assessment of techniques using Relaxo Footwear dataset considering monthly basis scenario with MSE and RMSE. The assessment of techniques with MSE is depicted in Fig. 8a. For 50\% training data, the MSE measured by FFO+MKSVM, GA-CNN, M-MI model, Deep LSTM, EHO, and proposed Rider-EHO based Deep-ConvLSTM are 207972.264, 126494.828, 89025.129, 84931.522, 61506.676, and 1274.588. Likewise, for 90\% data, the MSE measured by FFO+MKSVM, GA-CNN, M-MI model, Deep LSTM, EHO, and proposed Rider-EHO based Deep-ConvLSTM are 81332.992, 77380.833, 31816.548, 28424.865, 16968.819, and 336.285. The assessment of techniques with RMSE is depicted in Fig. 8b. For 50\% training data, the RMSE measured by FFO+MKSVM, GA-CNN, M-MI model, Deep LSTM, EHO, and proposed Rider-EHO based Deep-ConvLSTM are 456.040, 355.661, 298.371, 291.430, 248.005, and 35.701. Likewise, for 90\% data, the RMSE measured by FFO+MKSVM, GA-CNN, M-MI model, Deep LSTM, EHO, and proposed Rider-EHO based Deep-ConvLSTM are 285.189, 278.174, 178.372, 168.597, 130.264, and 18.338. 


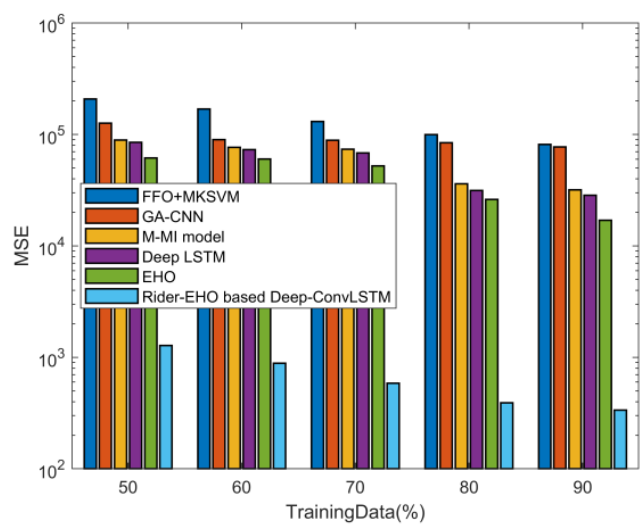

(a)

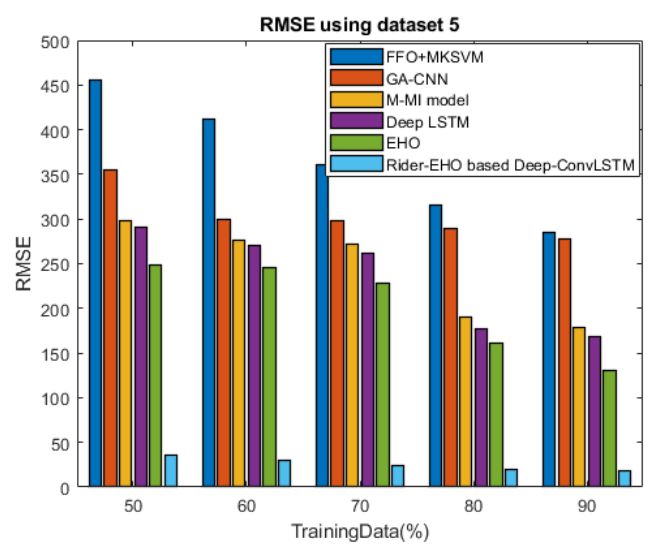

(b)

Fig. 8. Assessment of techniques using Relaxo Footwear dataset considering monthly basis scenario with a) MSE b) RMSE

4.4.6 Assessment with dataset-6

Fig. 9 presents the assessment of techniques using Relaxo Footwear dataset considering yearly basis scenario with MSE and RMSE. The assessment of techniques with MSE is depicted in Fig. 9a. For 50\% training data, the MSE measured by FFO+MKSVM, GA-CNN, M-MI model, Deep LSTM, EHO, and proposed Rider-EHO based DeepConvLSTM are 112696.409, 57425.375, 34279.311, 30410.691, 29921.674, and 11268.124. Likewise, for 90\% data, the MSE measured by FFO+MKSVM, GA-CNN, M-MI model, Deep LSTM, EHO, and proposed RiderEHO based Deep-ConvLSTM are 37075.898, 35269.354, 3800.855, 2105.128, 1968.611, and 554.895. The assessment of techniques with RMSE is depicted in Fig. 9b. For 50\% training data, the RMSE measured by FFO+MKSVM, GA-CNN, M-MI model, Deep LSTM, EHO, and proposed Rider-EHO based Deep-ConvLSTM are 335.703, 239.636, 185.147, 174.387, 172.979, and 106.151. Likewise, for $90 \%$ data, the RMSE measured by FFO+MKSVM, GA-CNN, M-MI model, Deep LSTM, EHO, and proposed Rider-EHO based Deep-ConvLSTM are $192.551,187.801,61.651,45.882,44.369$, and 23.556.

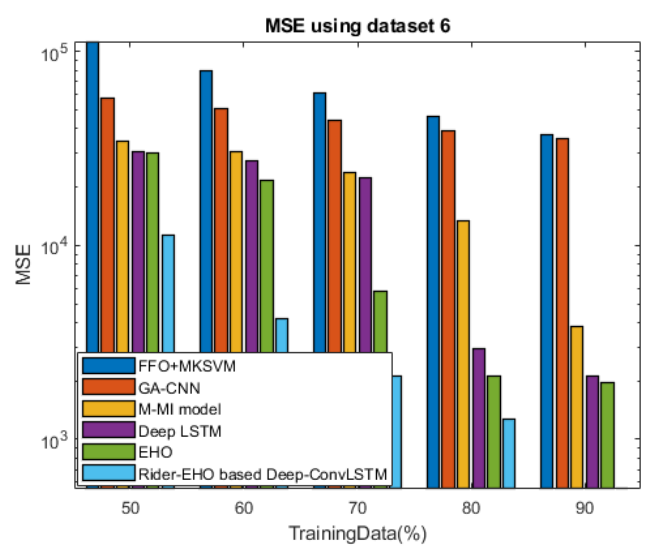

(a)

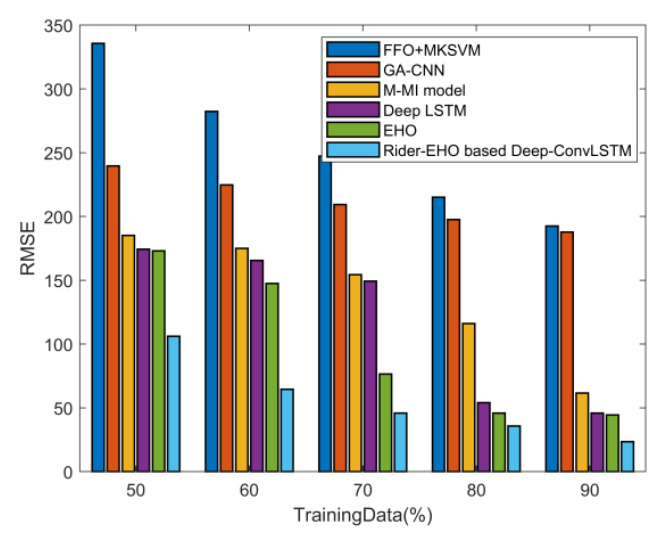

(b)

Fig. 9. Assessment of techniques using Relaxo Footwear dataset considering yearly basis scenario with a) MSE b) RMSE

\subsection{Comparative discussion}

Table 2 describes assessment of techniques considering MSE and RMSE using Reliance communication and Relaxo Footwear dataset by employing daily, monthly and yearly basis. Using dataset-1, the minimal MSE of 0.003 is evaluated by developed Rider-EHO based Deep-ConvLSTM while the MSE of remaining FFO+MKSVM, GA-CNN, M-MI model, Deep LSTM, EHO are 86.386, 0.377, 0.129, 0.033, and 0.013. The minimal RMSE of 0.059 is evaluated by proposed Rider-EHO based Deep-ConvLSTM whereas the RMSE measured by FFO+MKSVM, GA-CNN, M-MI model, Deep LSTM, EHO are 9.294, 0.614, 0.360, 0.182, and 0.116 . Using dataset-2, the minimal MSE of 44.435 is evaluated by proposed Rider-EHO based Deep-ConvLSTM while the minimal RMSE of 6.666. Using dataset-3, the smallest MSE of 50.396 and smallest RMSE of 7.099 is evaluated by proposed Rider-EHO based Deep-ConvLSTM. With dataset-4, the proposed Rider-EHO based Deep-ConvLSTM measured smallest MSE of 3.731 and smallest RMSE of 1.931. Using dataset-5, the proposed Rider-EHO based Deep-ConvLSTM measured smallest MSE of 336.285 and smallest RMSE of 18.338. With 
dataset-6, the proposed Rider-EHO based Deep-ConvLSTM measured smallest MSE of 554.895 and smallest RMSE of 23.556.

\begin{tabular}{|c|c|c|c|c|c|c|c|}
\hline Dataset & Metric & $\begin{array}{c}\text { FFO+ } \\
\text { MKSVM }\end{array}$ & GA-CNN & M-MI model & Deep LSTM & EHO & $\begin{array}{c}\text { Proposed Rider- } \\
\text { EHO based } \\
\text { Deep- } \\
\text { ConvLSTM } \\
\end{array}$ \\
\hline \multirow[t]{2}{*}{ Dataset-1 } & MSE & 86.386 & 0.377 & 0.129 & 0.033 & 0.013 & 0.003 \\
\hline & RMSE & 9.294 & 0.614 & 0.360 & 0.182 & 0.116 & 0.059 \\
\hline \multirow[t]{2}{*}{ Dataset-2 } & MSE & 779.248 & 440.134 & 150.028 & 117.319 & 83.308 & 44.435 \\
\hline & RMSE & 27.915 & 20.979 & 12.249 & 10.831 & 9.127 & 6.666 \\
\hline \multirow[t]{2}{*}{ Dataset-3 } & MSE & 8997.309 & 699.406 & 413.183 & 322.455 & 253.156 & 50.396 \\
\hline & RMSE & 94.854 & 26.446 & 20.327 & 17.957 & 15.911 & 7.099 \\
\hline \multirow{2}{*}{ Dataset-4 } & MSE & 127871.562 & 15644.667 & 170.773 & 142.793 & 86.504 & 3.731 \\
\hline & RMSE & 357.591 & 125.079 & 13.068 & 11.950 & 9.301 & 1.931 \\
\hline \multirow{2}{*}{ Dataset-5 } & MSE & 81332.992 & 77380.833 & 31816.548 & 28424.865 & 16968.819 & 336.285 \\
\hline & RMSE & 285.189 & 278.174 & 178.372 & 168.597 & 130.264 & 18.338 \\
\hline \multirow[t]{2}{*}{ Dataset-6 } & MSE & 37075.898 & 35269.354 & 3800.855 & 2105.128 & 1968.611 & 554.895 \\
\hline & RMSE & 192.551 & 187.801 & 61.651 & 45.882 & 44.369 & 23.556 \\
\hline
\end{tabular}

Table 2. Comparative analysis

\section{Conclusion}

This paper devises novel stock market prediction model considering an optimization assisted deep learning model. Initially, the review data of user and data are fed as an input to feature extraction phase. The mining of features is performed using the stock data and review data. From stock data, the technical indicators, such as APO, DMI, SMA, WILLR, TRIMA, and PPO are extracted. On review data, the stemming and stop word removal methods are adapted in order to extract the inappropriate words. Thereafter, SentiWordNet is employed for extracting the review-based features. These technical indicators along with opinion-based features represent the whole features and used for predicting stock data. The obtained features are modeled in a feature vector and subjected to DeepConvLSTM to predict movements of stock market. Here, the Deep-ConvLSTM training is carried out by employing a newly devised optimization method, namely Rider-EHO, which is generated by combining ROA and EHO. The developed Rider-EHO-based Deep-ConvLSTM attained improved performance in contrast to other methods with smallest MSE of 0.003 and smallest RMSE of 0.059 . In future, other datasets can be employed to further validate the efficiency of the model.

\section{References}

[1] Zhang X.; Qu, S.; Huang, J.; Fang, B.; Yu, P. (2018): Stock Market Prediction via Multi-Source Multiple Instance Learning, IEEE Access, 6, pp.50720 - 50728.

[2] Zhang, X.; Zhang, Y.; Wang, S.; Yao, Y.; Fanga, B.; Yu, P. S. (2018): Improving Stock Market Prediction via Heterogeneous Information Fusion, Knowledge-Based Systems, 143, pp. 236-247.

[3] Zhang, X.; Shi, J.; Wang, D.; Fang, B. (2018): Exploiting investors social network for stock prediction in China's market, Journal of Computational Science, 28, pp.294-303.

[4] Pang, X.; Zhou, Y.; Wang, P.; Lin, W.; Chang, V. (2018): An innovative neural network approach for stock market prediction, The Journal of Supercomputing, pp.1-21.

[5] Chandar, S. K. (2017): Stock market prediction using subtractive clustering for a neuro fuzzy hybrid approach, Cluster Computing, pp.18.

[6] Nayak, S. C.; Misra, B. B.; Behera, H. S. (2018): ACFLN: artificial chemical functional link network for prediction of stock market index, Evolving Systems, pp.1-26.

[7] Li, X.; Xie, H.; Wang, R.; Cai, Y.; Cao, J.; Wang, F.; Min, H.; Deng, X. (2016): Empirical analysis: stock market prediction via extreme learning machine, Neural Computing and Applications, 27(1), pp 67-78.

[8] Alam, M. M. G.; Baulkani. S. (2018): Local and global characteristics-based kernel hybridization to increase optimal support vector machine performance for stock market prediction, Knowledge and Information Systems, pp.1-30.

[9] Song, Y.; Lee, J. W.; Lee, J. (2018): A study on novel filtering and relationship between input-features and target-vectors in a deep learning model for stock price prediction, Applied Intelligence, pp.1-15.

[10] Senapati, M. R.; Das, S.; Mishra, S. (2018): A Novel Model for Stock Price Prediction Using Hybrid Neural Network, Journal of The Institution of Engineers (India): Series B, 99(6), pp.555-563.

[11] Fama, E. F. (1965): The behavior of stock-market prices, The journal of Business, 38(1), pp.34-105.

[12] Das, S. R.; Chen, M. Y. (2007): Yahoo! for amazon: Sentiment extraction from small talk on the web, Management science, 53(9), pp.1375-1388.

[13] Kim, I.M.; Park, S.K. (2009): The predictability of korean stock returns and volatility clock samples, The Korean Economic Association, 57(3), pp.195-221.

[14] Kim, S.D. (2012): Data mining tool for stock investors' decision support, The Journal of the Korea Contents Association, 12(2), pp.472482.

[15] Booth, A.; Gerding, E.; McGroarty, F. (2014): Automated trading with performance weighted random forests and seasonality, Expert Systems with Applications, 41(8), pp.3651-3661.

[16] Liu, H. C.; Lee Y. H.; Lee, M.C. (2009): Forecasting china stock markets volatility via GARCH models under skewed-GED distribution, Journal of Money, Investment and Banking, 7, pp.5-14. 
[17] George E. P. B.; Jenkins, G. M.; Reinsel, G. C.; Ljung, G. M. (2015): Time series analysis-forecasting and control, Holden-Day Inc., San Francisco.

[18] Osório, G. J. O; Matías, J. C. O.; Catalão, J. P. S. (2014): Hybrid evolutionary adaptive approach to predict electricity prices and wind power in the short term, proceedings of the Power Systems Computation Conference.

[19] Sumona, M.; Banerjee, S. (2012): Global optimization of an optical chaotic system by chaotic multi swarm particle swarm optimization", Expert Systems with Applications, 39(1), pp.917-924.

[20] Leung, M. T.; Daouk, H.; Chen, A.S. (2000): Forecasting stock indices: a comparison of classification and level estimation models, International Journal of Forecasting, 16(2), pp.173-190.

[21] Hu, C.; He, L. T. (2007): An application of interval methods to stock market forecasting, Reliable Computing, 13(5), pp.423-434.

[22] Chong, E.; Han, C.; Park, F. C. (2017): Deep Learning Networks for Stock Market Analysis and Prediction: Methodology, Data Representations, and Case Studies, Expert Systems with Applications, 83, pp.187-205.

[23] Binu, D.; Kariyappa, B. S. (2017): RideNN: A New Rider Optimization Algorithm-Based Neural Network for Fault Diagnosis in Analog Circuits, IEEE Transactions on Instrumentation and Measurement.

[24] Wang, G.; Deb. S.; Coelho, L. S. (2015): Elephant Herding Optimization, proceedings of the 3rd International Symposium on Computational and Business Intelligence.

[25] Song, X., Chen, K., Li, X., Sun, J., Hou, B., Cui, Y., Zhang, B., Xiong, G. and Wang, Z., 2020. Pedestrian trajectory prediction based on deep convolutional lstm network. IEEE Transactions on Intelligent Transportation Systems.

[26] Ghosh, M.; Kar, A. (2013): Unsupervised linguistic approach for sentiment classification from online reviews using SentiWordNet 3.0, Int J Eng Res Technol, 2(9).

[27] Chung, H.; Shin, K.S. (2020): Genetic algorithm-optimized multi-channel convolutional neural network for stock market prediction, Neural Computing and Applications, 32(12), pp.7897-7914.

[28] Gupta, A. (2020): Vertical Handover Using Mutated-Salp Swarm Optimization Algorithm Based On Radial Basis Function Neural Network In Heterogeneous Networks, Journal of Networking and Communication Systems, 3(3).

[29] Long, J.; Chen, Z.; He, W.; Wu, T.; Ren, J. (2020): An integrated framework of deep learning and knowledge graph for prediction of stock price trend: An application in Chinese stock exchange market, Applied Soft Computing, 4, pp.106205.

[30] Chowdhury, R.; Mahdy, M. R.; Alam, T. N.; Al Quaderi G. D.; Rahman, M. A. (2020): Predicting the stock price of frontier markets using modified Black-Scholes Option pricing model and machine learning, Physica A: Statistical Mechanics and its Applications, 7, pp. 124444

[31] Wen, M.; Li, P.; Zhang, L.; Chen, Y. (2019): Stock Market Trend Prediction Using High-Order Information of Time Series, IEEE Access, 7, pp.28299-308.

[32] Chen, Y.; Lin, W.; Wang, J. Z. (2019): A Dual-Attention-Based Stock Price Trend Prediction Model with Dual Features, IEEE Access, 7, pp.148047-58.

[33] Zhang, L.; Liu, N.; Yu, P. (2019): A novel instantaneous frequency algorithm and its application in stock index movement prediction, IEEE Journal of Selected Topics in Signal Processing, 6(4), pp.311-8.

[34] Wang, W.; Shi, Y.; Luo, R. (2019): Sparse Representation Based Approach to Prediction for Economic Time Series, IEEE Access, 7 , pp.20614-20618.

[35] Aithal, P. K.; Dinesh, A. U.; Geetha, M. (2019): Identifying Significant Macroeconomic Indicators for Indian Stock Markets, IEEE Access, 7, pp.143829-143840.

[36] Wang, Y.; Liu, H.; Guo, Q.; Xie, S.; Zhang, X. (2019): Stock Volatility Prediction by Hybrid Neural Network, IEEE Access, vol.7, pp.154524-154534.

[37] Das, S. R.; Mishra, D.; Rout, M. (2019): Stock market prediction using Firefly algorithm with evolutionary framework optimized feature reduction for OSELM method, Expert Systems with Applications, 4, pp.100016.

\section{Authors Profile}

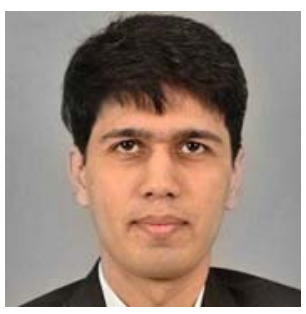

Jaymit B. Pandya, is currently pursuing Ph.D. in Computer/IT Engineering at Gujarat Technological University, Chandkheda, Gujarat, India. He has completed his B.E. from Shantital Shah Engineering College, Bhavnagar, Gujarat. He has completed his M.E. from Birla Vishvakarma Mahavidyalaya Engineering College, Vallabh Vidhyanagar, Gujarat. He is currently serving as a lecturer in Sir Bhavsinhji Polytechnic Institute, Bhavnagar, Gujarat. His research interests include image processing, machine learning, and deep learning.

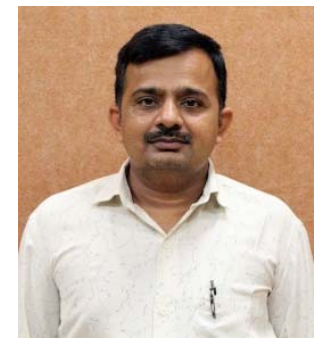

Prof. (Dr.) Udesang K. Jaliya, is a leading researcher \& academician in Computer Engineering and holds Ph.D. degree. He is currently working as an Assistant Professor in the Department of Computer Engineering at Birla Vishvakarma Mahavidyalaya Engineering College, Vallabh Vidhyanagar, Gujarat, India. He is a member of Computer Society of India. He has published 35 international articles. He has guided 28 research projects at master degree. He is currently guiding $4 \mathrm{Ph}$.D. students. His research interests include image processing, machine learning, and deep learning. 